\title{
Recent Advances in the Analysis of Extreme Metocean Events
}

\author{
Kevin Ewans, Sarawak Shell Bhd, Philip Jonathan, Shell Global Solutions
}

Copyright 2014, Offshore Technology Conference

This paper was prepared for presentation at the Offshore Technology Conference Asia held in Kuala Lumpur, Malaysia, 25-28 March 2014.

This paper was selected for presentation by an OTC program committee following review of information contained in an abstract submitted by the author(s). Contents of the paper have not been reviewed by the Offshore Technology Conference and are subject to correction by the author(s). The material does not necessarily reflect any position of the Offshore Technology Conference, its officers, or members. Electronic reproduction, distribution, or storage of any part of this paper without the written consent of the Offshore Technology Conference is prohibited. Permission to reproduce in print is restricted to an abstract of not more than 300 words; illustrations may not be copied. The abstract must contain conspicuous acknowledgment of OTC copyright.

\begin{abstract}
Accurate and reliable estimates of probabilities of rare, extreme metocean events are critical for optimal design of offshore facilities. They ensure facilities are neither over- nor under-designed, allowing target reliability levels to be achieved without undue conservatism. Engineering design requires metocean parameters to be specified with a return-period of 100 years, but often specification to a return period of 10,000 years is required. Modern hindcast data bases, typically used to derive extremal criteria, can be of limited extent, consisting of data for a few decades; hindcasts with periods of more than 50 years remain unusual. In addition, metocean criteria are often stratified by covariate - seasonality or directionality are common examples. Further, specification of joint occurrence of parameters at long return periods is necessary to avoid excessive conservatism, and such criteria may also need to be specified as functions of one or more covariates. Methods used by practitioners to meet these requirements are often somewhat adhoc, based on experience and intuition. In this paper we review recent applications which add statistic rigour and consistency to the estimation of design values. In particular, we present methods for maximising the benefit of limited data sets and deriving consistent extremal criteria with covariate, resulting in criteria that are consistent with respect to multiple covariates, including space, time and directionality.
\end{abstract}

\section{Introduction}

The ocean climate is a complex function of many factors. For example, the climate in the North Sea is dramatically different to that in the South China Sea, and within an ocean basin the climate can be significantly different from one location to another, depending on a given location's proximity to storm tracks, the coast, and water depth. Even at a given location, the climate will vary depending on the season and on the directionality of the prevailing weather. In the same way, the extremal ocean climate is a function of these variables, and these variables must be considered and assessed when developing extremal design criteria for the design of offshore facilities.

Fortunately, hindcast databases are usually available for the determination of ocean parameter extremes for design purposes, and these data contain all the inherent effects associated with the variability of a parameter, and it is possible to take these effects or covariates into account when determining design criteria. However, covariate effects are often ignored in practice because of the complexity of extreme value modelling with covariates, but it is important that effects of covariates are adequately modelled.

Improvements in structural reliability for no additional cost are achievable in principle if covariate effects are incorporated in the specification of design criteria in general e.g. by designing structures with different strengths in different directions or minimising the number of mooring lines by optimising them with direction, but more importantly, estimates for extreme quantiles obtained from modelling that ignores covariate effects will not be the same as those obtained from models incorporating covariate effects, and more accurate extremal modelling will result if the covariates are included. For example, offshore Sarawak, the wave climate, including the extreme events, is dominated by the southeast and northwest monsoon. These are not only associated with different directions, but they also occur at different times of the year; and in particular, the extreme sea states associated with these two monsoons have different probability distributions, the extremal model should properly account for this variation.

Thus, the modelling should incorporate the effects associated with the variability of the probability of extremes as a function of season and of direction (as in Feld et al. 2014), and there are numerous examples in the literature. Coles and Walshaw (1994) develop a directional model for extreme wind speed. Coles and Casson (1998) propose an extreme value model for 
hurricane wind speed with spatially-varying parameters. Butler et al. (2007) characterize changes in the occurrence and severity of storm surge events in the southern and central North Sea over the period 1955-2000. Mendez et al. (2008) introduce a seasonal peaks-over-threshold model for significant wave height. Ruggiero et al. (2010) examine increasing wave heights and extreme value projections for the U.S. Pacific Northwest. Kyselý et al. (2010) estimates extremes in climate change simulations using the peaks over threshold method with non-stationary threshold. Northrop and Jonathan (2011) discuss threshold modelling of spatially dependent non-stationary extremes with application to hurricane-induced significant wave height.

In previous work (e.g Jonathan and Ewans (2007), Ewans and Jonathan (2008), Jonathan et al. (2008)) it has been shown that omnidirectional design criteria derived from a model that adequately incorporates directional covariate effects can be materially different from a model which ignores those effects. Directional storm peaks $H_{s 100}$ derived from a directional model can be heavier tailed than that derived from a direction-independent approach, indicating that large values of storm peak $H_{s}$ are more likely than we might anticipate were we to base our beliefs on estimates which ignore directionality. Similar effects have been demonstrated for seasonal covariates (e.g. Anderson et al. 2001, Jonathan et al. 2008). So even when the objective is to determine the annual exceedance probability of a single parameter, such as wind speed, at a given location, it is important to include the effects of seasonality and direction. These effects will vary as a function of location, resulting in variations in the exceedance probability of the parameter of interest, which can be significant even for neighbouring points.

In addition, the occurrence probability of one parameter given an extreme occurrence of another is in general a function of covariates. For example, the probability of occurrence of wave spectral peak period, given an extreme value of significant wave height, will vary as a function of season and direction (Ewans and Jonathan, 2008 and Jonathan and Ewans 2011), and for example joint criteria that accounts for this dependency is important for the design of the mooring configuration of an FPSO.

The conditional extremes model of Heffernan and Tawn (2004) provides a straight forward framework for estimating multivariate extremal dependence in the absence of covariates. The approach uses an asymptotic argument which conditions on one component of a random vector and finds the limiting conditional distribution of the remaining components as the conditioning variable becomes large.

A number of applications and refinements of the conditional extremes approach have been reported. Keef et al. (2009) examine spatial risk assessment for extreme river flows using the conditional extremes model. Keef et al. (2013a) propose a variant of the conditional extremes model in which marginal transformation to Laplace rather than Gumbel scale is performed. Keef et al. (2013b) propose additional constraints within the conditional extremes model formulation, particularly relevant for negatively associated variables. Gilleland et al. (2013) use the conditional extremes model to estimate joint extremes of largescale indicators for severe weather.

There are numerous other approaches, including the popular empirical method of Haver and Nyhus (1986), and the first order reliability method (FORM) and inverse-FORM (I-FORM) methodologies (e.g. Winterstein et al. 1993 and Winterstein et al. 1999), both of which attempt to characterise the marginal and conditional distributions of oceanographic variables.

Jonathan et al. (2013) incorporated the effect of covariates in the conditional extremes model of Heffernan and Tawn (2004), which has been further extended to involve a common general-purpose penalised spline representation of model parameters with respect to multidimensional covariates (Jonathan et al., 2014).

This paper describes recent developments in methods for estimating extremal metocean design criteria, both for the marginal and joint cases. The methods are described in the next section. Several examples of the application of these methods are given in the Results section. Then we finish with some final remarks in the Discussion section.

\section{Method}

\section{Marginal Estimates with Covariates}

The most commonly used method for developing extreme design criteria is to follow the peaks over threshold (POT) approach for selecting extremes in the data, followed by fitting the extreme values with a generalized Pareto distribution (GPD) and computing extreme quantiles assuming the occurrences of threshold exceedances is a Poisson process. This is preferred over the perhaps more intuitive approach of selecting annual maxima (AM) data and then fitting these data with a generalized extreme value distribution (GEV) from which extreme quantiles can be estimated directly, as more extremal data are available from the POT method than from the AM method. Both the GEV and GPD methods are based on asymptotic arguments and excellent descriptions of both can be found in Coles (2007).

Both the POT and the AM methods can be exteneded to account for one or more covariates. Unfortunately, commonly used practices have often led to inconsistencies in design criteria, such as that the probability of exceedance of a given significant wave height when calculated from the directional criteria is different from that obtained from omnidirectional criteria. A 
discussion of this problem is given by Forristall (2004). There are however techniques involving for example Fourier-based or spline-based approaches for modelling covariate that are rigorous and provide consistent covariate and omni-covariate criteria.

We employ a spline based methodology to incorporate spatial, directional, temporal and other covariate effects in extreme value models for environmental variables such as storm severity. For storm peak signicant wave height events, the approach uses quantile regression to estimate a suitable extremal threshold, a Poisson process model for the rate of occurrence of threshold exceedances, and a generalised Pareto model for size of threshold exceedances. Multidimensional covariate effects are incorporated at each stage using penalised (tensor products of) B-splines to give smooth model parameter variation as a function of multiple covariates. Optimal smoothing penalties are selected using cross-validation, and model uncertainty is quantified using a bootstrap re-sampling procedure. We now discuss each of these components.

By way of example, let us say our objective is to estimate design criteria for individual locations within a spatial neighbourhood, accounting for spatial and storm directional variability of extremal characteristics. We model storm peak significant wave height, namely the largest value of significant wave height observed at each location during the period of a storm event.

We assume that each storm event is observed at all locations within the neighbourhood under consideration. For a sample $\left\{\dot{z}_{i}\right\}_{i=1}^{\dot{n}}$ of $\dot{n}$ storm peak significant wave heights (henceforth $H_{s}^{s p}$ observed at locations $\left\{\dot{x}_{i}, \dot{y}_{i}\right\}_{i=1}^{\dot{n}}$ with dominant wave directions $\left\{\dot{\theta}_{i}\right\}_{i=1}^{\dot{n}}$ at storm peak $H_{s}$ (henceforth "storm directions"), we proceed using the peaks over threshold approach as follows.

Extreme value threshold: We first estimate a threshold function - above which observations $\dot{z}$ are assumed to be extreme. The threshold varies smoothly as a function of covariates $(\phi \triangleq \phi(\theta, x, y))$ and is estimated using quantile regression. We retain the set of $n$ threshold exceedances $\left\{z_{i}\right\}_{i=1}^{\dot{n}}$ observed at locations $\left\{x_{i}, y_{i}\right\}_{i=1}^{\dot{n}}$ with storm peak directions $\left\{\theta_{i}\right\}_{i=1}^{\dot{n}}=1$ for further modelling.

Rate of threshold exceedance: We next estimate the rate of occurrence $\rho$ of threshold exceedance using a Poisson process model with Poisson rate $\rho \triangleq \rho(\theta, x, y)$.

Size of threshold exceedance: We estimate the size of occurrence of threshold exceedance using a generalised GPD. The GPD shape and scale parameters $\xi$ and $\sigma$ are also assumed to vary smoothly as functions of covariates.

The smoothness of the parameters is achieved for the multi-dimensional covariate domain by developing a basis matrix, which is a Kronecker product of marginal basis matrices. For example, in the case of a spatio-direction model, our basis matrix would be

$$
B=B_{\theta} \otimes B_{x} \otimes B_{y}
$$

Where for example $B_{x}$ is B-spline basis matrix for direction, and $B_{\theta}$ is the B-spline basis matrix periodic in direction.

This approach to extreme value modelling follows that of Chavez-Demoulin and Davison (2005) and is equivalent to direct estimation of a non-homogeneous Poisson point process model (e.g., Dixon et al. 1998, Jonathan and Ewans 2013). Details of the parameter estimation can be found in Jonathan et al. (2014)

Return value $z_{T}$ of storm peak significant wave height corresponding to some return period $T$, expressed in years, can be evaluated in terms of estimates for model parameters $\phi, \rho, \xi$, and $\sigma$. For any choice of covariates $\theta, x, y$, the return value is given by

$$
z_{T}=\phi-\frac{\sigma}{\xi}\left(1+\frac{1}{\rho}\left(\log \left(1-\frac{1}{T}\right)\right)^{-\xi}\right)
$$

where all of $\phi, \rho, \xi$ and $\sigma$ are understood to be functions of $\theta, x, y$, and $\rho$ is expressed as an annual rate of threshold exceedance per location per 1 -degree storm direction. Thus, $z_{100}$ corresponds to the 100 -year return value, often denoted by $H_{s 100}$. Interpretation of the value of $z_{T}$ should be undertaken with considerable care. For example, in the current spatiodirectional case, the value of $z_{100}(\theta, x, y)$ corresponds to the 100 -year return value for storm peak significant wave height at location $(x, y)$ for storm directions within a 1-degree directional sector centred on direction $\theta$.

For estimation of directional design values per location, simulation under the fitted model incorporating the effects of storm directional dissipation is a better alternative, introduced by Jonathan and Ewans (2007). The directional dissipation $\zeta(\theta)$ of a storm, for direction $\theta$, is the value of significant wave height, expressed as a fraction of storm peak significant wave height, for that $\theta$. For a given directional sector $S$, therefore, the directional dissipation $\zeta(S)$ of a storm is the largest impact of the storm in $S$, expressed as a fraction of the storm peak significant wave height. Directional dissipation captures the effects on a directional sector of storms whose peaks lie outside the sector. Incorporating directional dissipation increases return values in general, for all directional sectors other that the omni-directional (which already includes the effects of all storms). 
Uncertainties are estimated by bootstrapping the extremal data set and repeating the analysis for each sample.

Examples of directional design values and estimated from simulation are given in the next section. The procedure for simulation is given in Jonathan et al. (2014).

\section{Conditional Extremes with Covariate}

The conditional extremes model of Heffernan and Tawn (2004) provides a framework for estimating multivariate extremal dependence in the absence of covariates. The approach uses an asymptotic argument which conditions on one component of a random vector and finds the limiting conditional distribution of the remaining components as the conditioning variable becomes large.

The conditional extremes model of Heffernan and Tawn (2004) of random variables $\dot{X}_{1}$ and $\dot{X}_{2}$, involves

(a) performing a marginal POT analysis for each variate,

(b) fitting the threshold exceedances with a GPD,

(c) transforming the $\dot{X}_{1}$ and $\dot{X}_{2}$ in turn to Gumbel scale (to $X_{1}$ and $X_{2}$ ) using the probability integral transform,

(d) estimating conditional extremes models for $X_{2} \mid X_{1}$ (and $\dot{X}_{1} \mid \dot{X}_{2}$ ) of the form

$$
\left(X_{2} \mid X_{1}=x\right)=\alpha x+x^{\beta} Z \text { for } x>u
$$

for an appropriate threshold $u$, where $\alpha \in(0,1]$ is the scale parameter, and $\beta \in(-\infty, 1]$ is the shape parameter, and $Z$ is a random variable, independent of $X$, converging with increasing $x$ to a non-degenerate limiting distribution, $G$ (which is assumed Gaussian for model fitting purposes only).

(e) retaining the estimated model parameters and residuals

(f) simulating joint extremes $\left\{X_{1}, X_{2}\right\}$ on the standard Gumbel scale under the model, assuming

$$
\hat{z}_{i}=\frac{y_{i}-\hat{\alpha} x_{i}}{x_{i}^{\beta}} \text { for } i=1,2, \ldots
$$

and finally

(g) transform realisations to the original scale using the probability integral transform.

The conditional extremes model is extended to incorporate covariates, $\theta$, in a similar way to the marginal distributions described above. Accordingly, the covariate dependence of the threshold and marginal GPD are defined in terms of a basis matrix consisting of Kronecker product of the respective covariate B-splines, and the GPDs are transformed into Gumbel scales using the probability integral transform, retaining the covariate dependence.

For positively dependent random variables $X_{1}(\theta), X_{2}(\theta)$ with standard Gumbel marginal distributions for any $\theta$, we extend the asymptotic argument Heffernan and Tawn (2004) expression becomes

$$
\left(X_{2}(\theta) \mid X_{1}(\theta)=x\right)=\alpha(\theta) x+x^{\beta(\theta)} Z(\theta)
$$

Extended forms are available for potentially negatively dependent variables.

Details of the model and the estimation procedure can be found in Jonathan et al. (2014).

\section{Results}

\section{Marginal Estimates with Covariates}

In this section we provide examples of the application of the methods outlined above for estimating extremes with covariates.

\section{Directional-seasonal Analysis}

In the first example, a non-stationary extreme value analysis is used to estimate the distributions of storm peak significant wave height $H_{s}^{s p}$ to arbitrary long return periods. The approach exploits recent advances in extreme value analysis with multidimensional covariates (described in the previous section) to characterise return value characteristics for $H_{s}^{s p}$ with direction and season (for the storm peak sea state only), for a location in the North Sea. The storm peak significant wave heights $H_{s}^{s p}$ are plotted in Figure 1, in the form of scatter plots with respect to storm direction $\theta^{s p}$ (upper panel) and storm season $\phi^{s p}$ (lower panel). The plots show clearly the variability in the occurrences of extreme $H_{s}^{s p}$ with direction and season. 

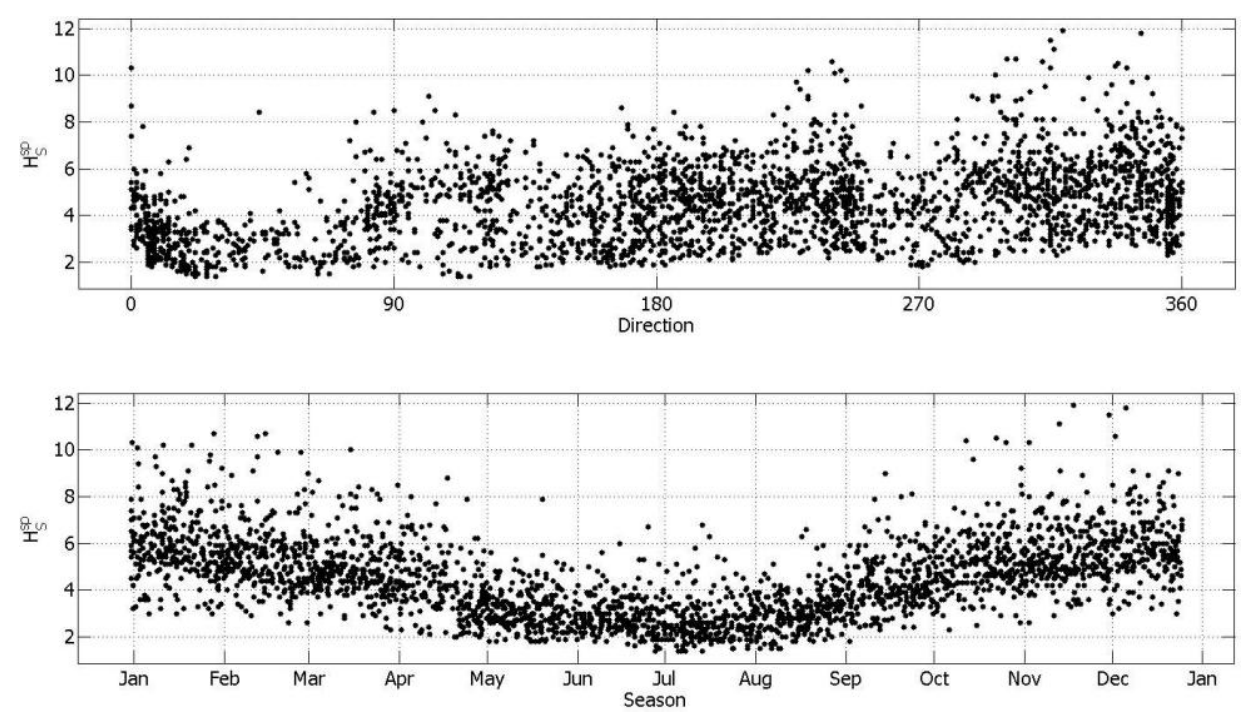

Figure 1. Storm peak significant wave height $H_{s}^{s p}$ on storm direction $\theta^{s p}$ (upper panel) and storm season $\phi^{s p}$ (lower panel). After Feld et al. (2014).

The analysis involves estimation of the model parameters as a function of covariate. By way of example, the estimates for the scale parameter of the GPD are given in Figure 2. The greatest directional variability occurs in the winter months, for winter storms emanating from directions in $[270,360)$.

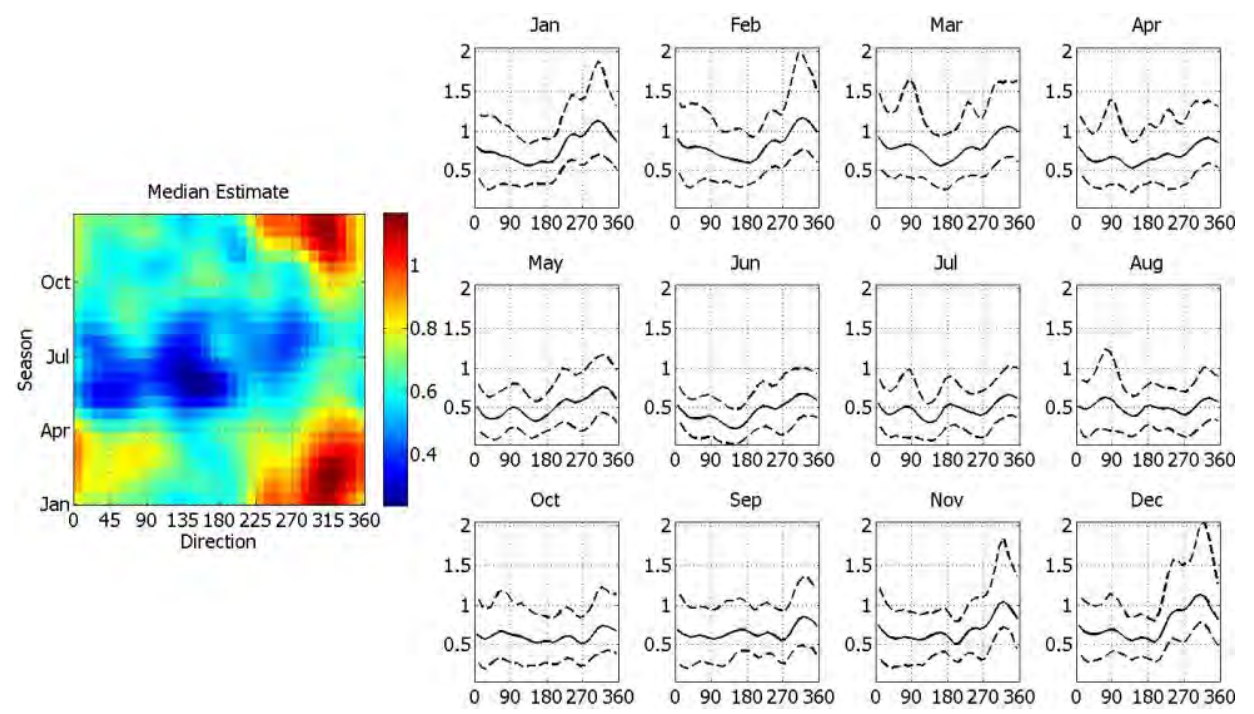

Figure 2. Directional-seasonal parameter plot for generalized Pareto scale, $\sigma$. The left-hand panel shows the bootstrap median scale on $\theta^{s p}$ and $\phi^{s p}$. The right hand panel shows 12 monthly directional scales in terms of bootstrap median (solid) and $95 \%$ bootstrap uncertainty band (dashed). After Feld et al. (2014).

These estimates allow return values corresponding to the return period $P$ of interest to be estimated by simulation under the model described above (details can be found in Jonathan et al., 2014). Empirical cumulative distribution functions for $H_{s}^{s p}$ are then trivially estimated by sorting the values for arbitrary combinations of covariate bins. In this way, for example, cumulative distribution functions for directional return values each month of the year, or seasonal return values for directional octants can be estimated. By retaining only maxima over all covariate bins, omni-directional omniseasonal are obtained. Importantly, since realisations based on models from different bootstrap resamples of the original sample are used, the resulting cumulative distribution functions incorporate both the (aleatory) inherent randomness of return values and the extra (epistemic) uncertainty introduced by model parameter estimation from a sample of data.

Figure 3 shows cumulative distribution functions (cdfs) for 100-year $H_{s}^{s p}$ from simulation under the directional-seasonal model, incorporating uncertainty in parameter estimation using bootstrap resampling as explained above. Upper panel shows cdfs for directional octants and lower panel for months of year. The common omni-directional omni-seasonal cdf is shown in both panels (in black). It is clear that the severest storms come from the north - west in winter months. The median omni - 
directional omni - seasonal 100 - year storm peak value is approximately $12.2 \mathrm{~m}$.
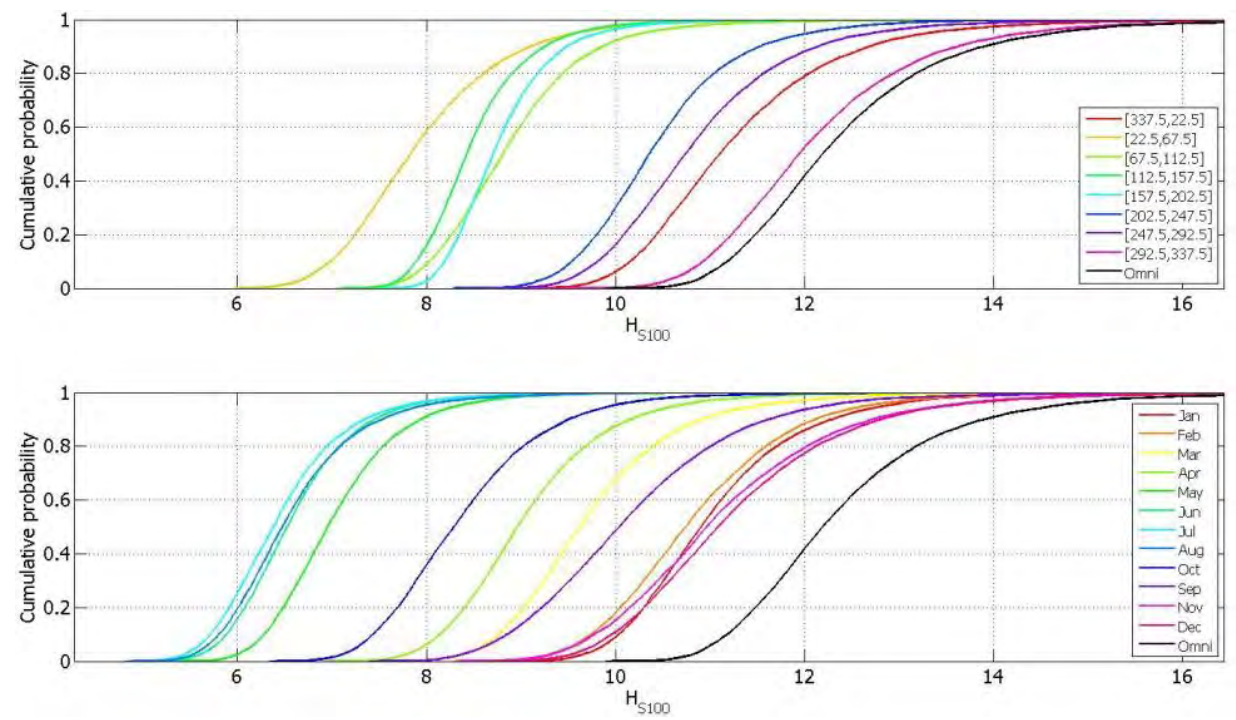

Figure 3. Cumulative distribution functions (cdfs) for 100-year $H_{s}^{s p}$ from simulation under the directional-seasonal model, incorporating uncertainty in parameter estimation using bootstrap resampling. Upper panel shows cdfs for directional octants and lower panel for months of year. The common omnidirectional omni-seasonal cdf is shown in both panels (in black). After Feld et al. (2014).

Figure 4 shows return value plots for $H_{s}^{s p}$. There are obvious and statistically significant differences between return values for different directions and seasons. It is important to note that all omni-directional and omni-seasonal estimates here are calculated from the directional-seasonal model; estimating these from models which ignore directional and seasonal variation in extremes would be inappropriate.
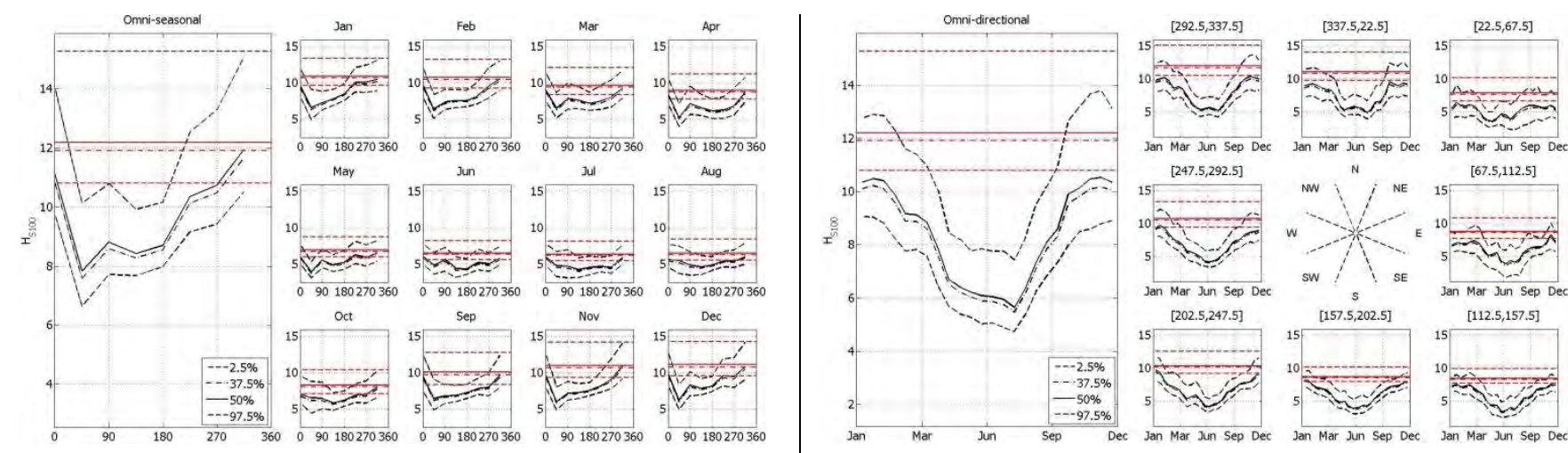

Figure 4. Directional-seasonal return value plot for 100-year $H_{s}^{s p}$. The left-hand panel shows omni-seasonal return values on wave direction $\theta$, in terms of directional octant median (solid black), most-probable (dot-dashed black), 2.5\%ile and 97.5\%ile (both dashed black) and the corresponding omni-directional omni-seasonal estimates. 12 monthly directional octant return values (in black) in terms of median (solid), most-probable (dot-dashed), 2.5\%ile and 97.5\%ile (both dashed) are also show, together with the corresponding omni-directional estimates (in red). The right-hand panel also shows return value plots for $H_{s}^{s p}$. But now the left-hand panel shows omni-directional return values on wave season $\phi$, in terms of monthly median (solid black), most-probable (dot-dashed black), 2.5\% ile and 97.5\%ile (both dashed black) and the corresponding omni-directional omniseasonal estimates (in red, common to Figure 4). The right hand panel shows seasonal return values for directional octants in terms of median (solid), most-probable (dot-dashed), 2.5\%ile and 97.5\%ile (both dashed). Also shown are the corresponding omni-seasonal estimates (in red). After Feld et al. (2014).

An example of a spatio-direction extremal analysis is given in Figure 5, which shows median 100-year return values, $H_{s}^{s p}$, for a region on the north west continental shelf of Western Australia. The extremal data are selected from a storm hindcast study involving 6156 hindcast storm events at 1089 locations on a 33 x 33 regular grid over the North West Shelf of Western Australia for the period 1970-2007. Both omni-directional return values and return values corresponding to directional sectors 
centred on the cardinal and inter-cardinal directions are given in Figure 5. In this case, a penalised B-splines formulation has been used to characterise smooth variation of extreme value parameters spatially and directionally. An index set of 32 directional bins x 33 longitude bins x 33 latitude bins. Cubic splines are used for each dimension, the directional spline basis being the only periodic. Longitude and latitude domains are characterised by 15 evenly spaced knots, and the directional domain by 32 evenly spaced knots. Therefore, a total of $32 \times 18 \times 18$ spline parameters need to be estimated. Directional dissipation is not included in Figure 5 results. Details of the analysis can be found in Jonathan et al. (2014).

The omni-directional plot (left) in Figure 5 shows generally lower return values in the south east corner, which is nearest land, The right hand set of eight directional sector plots indicate that the highest return values at most locations are generally from the east and north east and that return values are generally lower for storms from the west.

It is noticeable that the contours in Figure 5 are somewhat striated. This corresponds to the individual paths of tropical cyclones in the data set. There were 77 tropical storms hindcast, but the results show that this is really too few to provide a smooth extremal picture across this 4 degree square region. Given a tropical cyclone climatology corresponding to many multiples of this hindcast duration, we would expect a representation of the extremes as given in Figure 5 to vary smoothly over space. Nevertheless, the application of a spatial spline analysis has maximized use of the limited data set in that an estimate it any given point over the 4 degree square has been improved by virtue of the spatial smoothing provided by the splines.
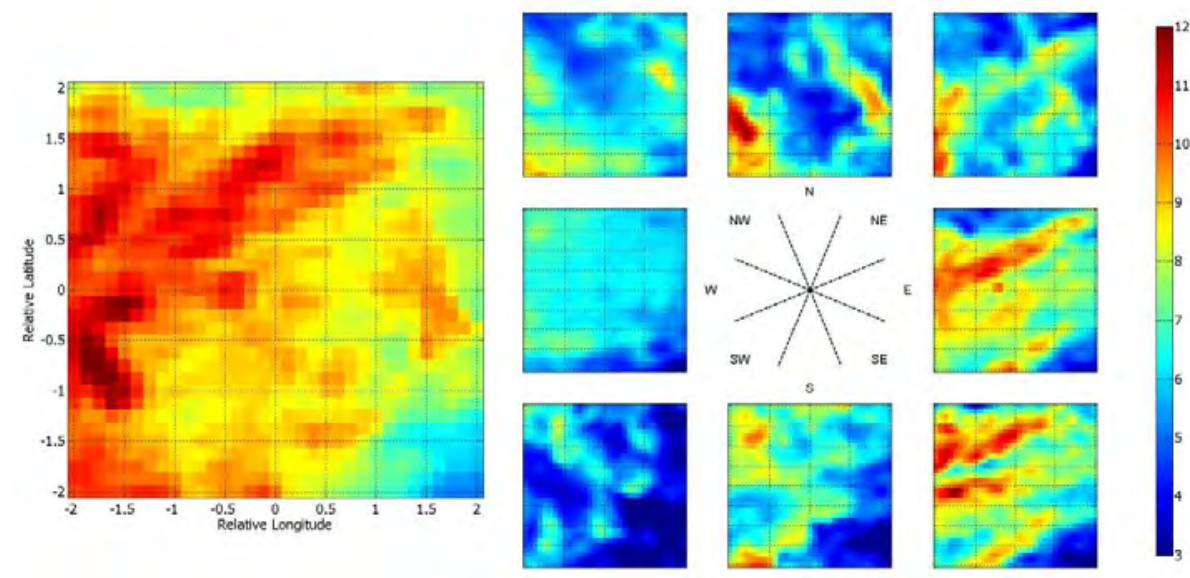

Figure 5: 100-year return values, $H_{s}^{s p}$. The left-hand plot shows median omni-directional return values for each of the 1089 locations. The 8 right-hand plots show median directional sector $H_{s}^{s p}$ per location for 8 directional octants centred (from left to right, top to bottom) on storms from NW, N, and NE; W and E, SW, S and SE respectively, and as indicated by the sectors in the centre schematic. Colour scale in metres. After Jonathan et al. (2014).

A particular application of the non-stationary covariate analyes is the provision of campaign-based criteria. By this we mean operations involving structures that are deployed for a specific duration, typically less than 12 months but which must meet a given environmental loading reliability. An example would be the deployment of a jack-up drilling rig for say a 6-month drilling campaign. Figure 6 shows 10-year and 50-year $H_{s}^{s p}$ criteria corresponding to a 6-month period, starting on any given date in the year, for a location in the South China Sea. The estimates have been derived from directional-seasonal analysis. The dashed horizontal lines are the all-year values.

Figure 6 shows clearly that the best time to start the campaign is mid-December. If the campaign commences then, the 50-year return period $H_{s}^{s p}$ for the 6-month exposure period is only $8.5 \mathrm{~m}$, whereas a jack-up rig would be required to comply with a 50year $H_{s}^{s p}$ of $11.2 \mathrm{~m}$. 


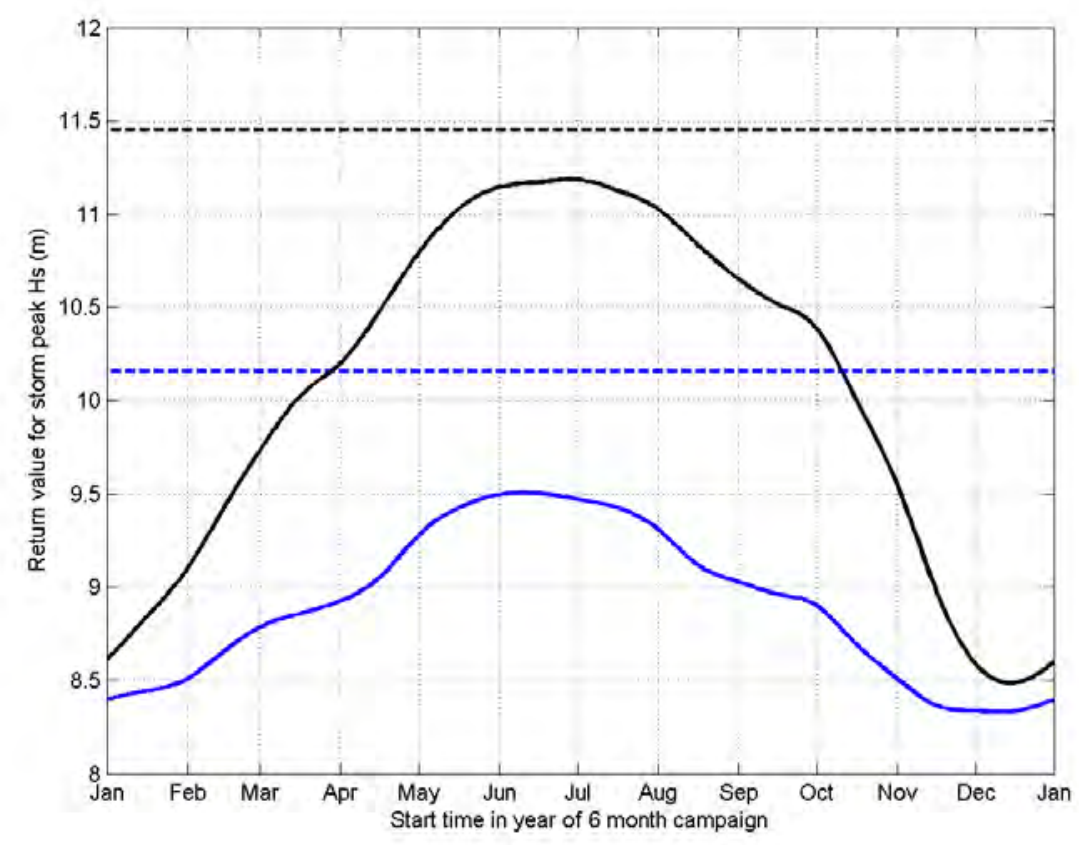

Figure 6: Return-period $H_{s}^{s p}$ values for a six month campaign as a function of start date (continuous), and the all-year values (dashed). Black: 50-year return-period, blue: 10-year return-period.

\section{Conditional Extremes with Covariate}

An example of the application of the method for estimating conditional extremes with covariate are the results for hindcast storm peak significant wave height, $H_{s}^{s p}$, and associated $T_{P}$ in the northern North Sea reported by Ewans et al. (2014). The objective is to model the distribution of $T_{P}$ for large $H_{s}^{s p}$ as a function of storm direction. The location is particularly useful for application of the model as the wave field has identifiable characteristics for various directional sectors, as can be seen in Figures. 7 and 8. Storms with the largest sea states are those occurring in the north, south, and southwest-west sectors; less severe sea states are associated with storms from the northwest sector; and virtually no storms occur that cause waves from the easterly sector. Further, it can be seen in Figure 7 that storm peak sea states from the northwest and southwest-west sectors are associated with the longest $T_{P}$ values. These characteristics should be evident in the conditional extremes modelling and can serve as an indicator of the success of the modelling. The joint distribution of $H_{S}^{s p}$ and $T_{P}$ below the threshold is modelled using quantile regression.

Conditional $T_{P}$ values corresponding to $H_{S}^{s p}$ values with exceedance probability of 0.01 are illustrated in Figure 8 (right). The inner (black and white) dotted curves, drawn on the same scale, illustrate estimates of the $H_{s}^{s p}$ return value. The inner white dotted curve is an estimate for the directional variation of the $H_{s}^{s p}$ return value. For comparison the inner black dotted curve is an estimate for the same return value ignoring directional effects. The influence of longer fetches from south (in particular), the Atlantic and Norwegian Sea are visible.

The outer (black and white) curves, drawn on the same scale, illustrate estimates for return values of $T_{P}$ conditional on exceedances of the corresponding $H_{s}^{s p}$ value. Solid lines represent median values, and dashed lines $95 \%$ uncertainty bands, incorporating (white) or ignoring (black) directional effects. The results clearly show increased associated periods from the Atlantic and Norwegian sectors, as expected. When directionality is ignored, associated $T_{P}$ values are underestimated for some sectors and overestimated for others. The importance of this difference for design can be seen in the response of a simple system with a transfer function characteristic of the roll or heave of a floating system with a natural period of around $17 \mathrm{~s}$. Response is over-estimated by more than $30 \%$ in directional sectors with short fetches, but under-estimated by as much as $20 \%$ in sectors with long fetches, particularly the Atlantic sector (see Jonathan et al., 2013). 

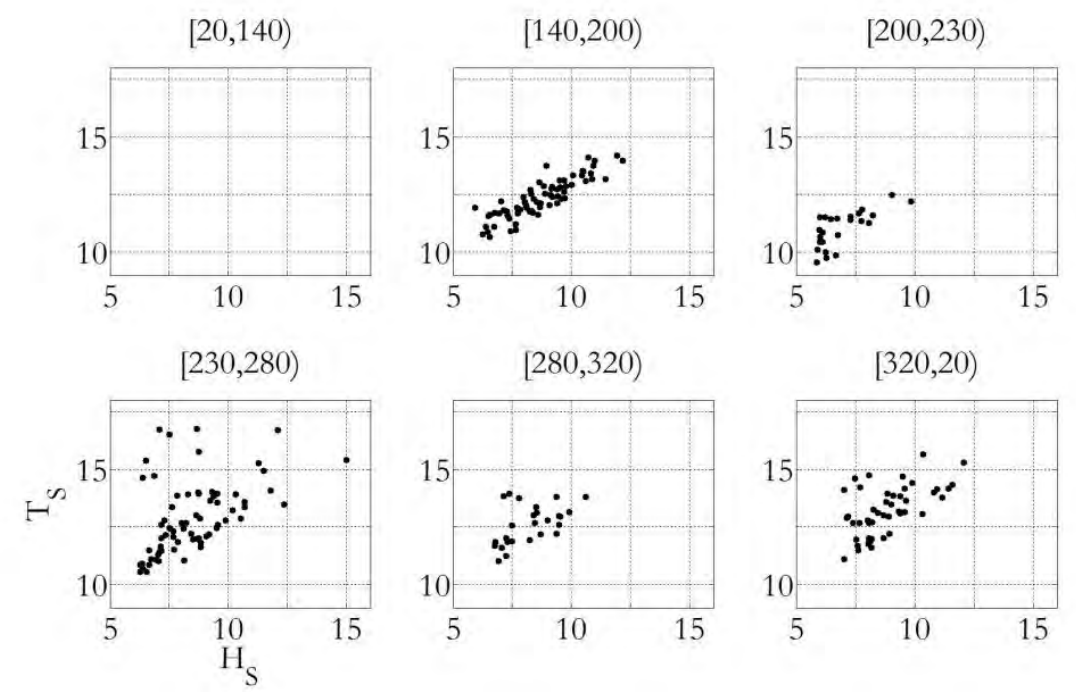

Figure 7. Scatter plots of associated $T_{P}$ against storm peak $H_{S}^{s p}$ for different directions of arrival.
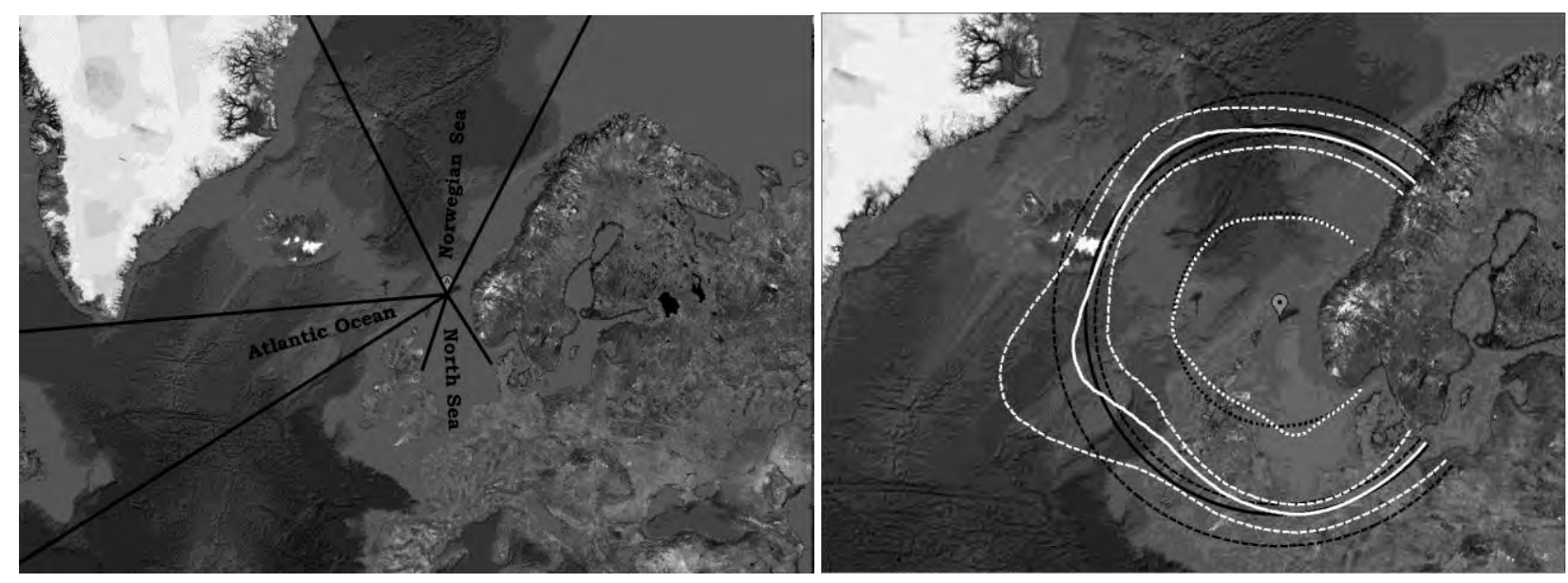

Figure 8. Left: Northern North Sea location and directional sectors with distinctive wave characteristics. Right: Return values of $H_{S}^{S p}$ (inner circle) and associated conditional values of $T_{P}$ (outer circle). Inner dashed lines (on common scale): storm peak $H_{s}^{s p}$ with non-exceedance probability 0.99 (in 34 years), with (white) and without (black) directional effects. Outer solid lines (on common scale): median associated $T_{P}$ with (white) and without (black) directional effects; outer dashed lines give corresponding $2.5 \%$ and $97.5 \%$ percentile values for associated $T_{P}$.

\section{Discussion}

Extreme value analysis is inherently different to other empirical modelling, in that estimation of tails (rather than bodies) of distributions, and extrapolation beyond the sample (rather than interpolation within) is demanded. Intuition accumulated from other areas of empirical modelling can be misleading. This paper outlines recent methods for improved extreme value modelling in ocean engineering, primarily through improved consideration of covariates, such as direction, season, and space. Incorporation of covariate effects will improve model fits in general, and reduce modelling uncertainty. Suitable tools, based on spline methods, have been developed that enable rigorous estimates of extreme values stratified by a particular or a combination of covariates.

Regarding modelling of multivariate extremes, effective modelling will improve the specification of design conditions (e.g. for significant wave height and associated values for other variables). Approaches such as the conditional model of Heffernan and Tawn (2004) are easily implemented, and provide generalisations of methods such as I-FORM (e.g. Winterstein et al., 1993). The conditional model is easily extended to incorporate covariates, following the methods used for marginals.

Finally, modelling spatial dependence well will provide single extreme value models applicable to complete ocean basins, avoiding the need for site pooling. Once the model is established, the metocean engineer may not ever need to perform extreme value analysis in principle; at the very least the basin model would provide a reliable first estimate for establishing site-specific criteria 


\section{References}

Anderson, C.W., Carter, D.J.T., and P.D. Cotton. 2001. Wave climate variability and impact on offshore design extremes. Report commissioned from the University of Sheffield and Satellite Observing Systems for Shell International.

Chavez-Demoulin, V., and A.C. Davison, 2005. Generalized additive modelling of sample extremes. J. Roy. Statist. Soc. Series C: Applied Statistics, 54, 207-2005.

Butler, A., Heffernan, J. E., Tawn, J. A., and R.A. Flather, 2007. Trend estimation in extremes of synthetic north sea surges. J. Roy. Statist. Soc. C 56, 395-414.

Coles, S., 2007. An introduction to statistical modeling of extreme values. Springer. $4^{\text {th }}$ Ed.

Coles, S., and D. Walshaw, 1994. Directional modelling of extreme wind speeds. Applied Statistics 43, 139-157.

Coles, S. G., and E. Casson, 1998. Extreme value modelling of hurricane wind speeds. Structural Safety 20, $283-296$.

Dixon, J.M., Tawn, J.A., and J. M. Vassie, 1998. Spatial modelling of extreme sea-levels. Environmetrics, 9, $283-301$.

Eilers, P H C, and B D Marx, 2010. Splines, knots and penalties. Wiley Interscience Reviews: Computational Statistics, 2:637653.

Ewans, K.C., and P. Jonathan, 2008. The effect of directionality on Northern North Sea extreme wave design criteria, $J$. Offshore Mechanics and Arctic Engineering, 130 (4), 10.

Ewans, K., and P. Jonathan, 2014. Evaluating environmental joint extremes for the offshore industry. Journal of Marine Systems, 130, 124-130.

Feld, G., Randell, D., Wu, Y., Ewans, K., and P. Jonathan. 2014. Estimation of storm peak and intra-storm directionalseasonal design conditions in the North Sea. OMAE2014-23157, Proc. 33nd Conf. Offshore Mech. Arct. Eng. (under review 2014, preprint at www.lancs.ac.uk/ jonathan).

Forristall, G.Z., 2004. On the use of directional wave criteria. Journal of Waterway, Port, Coastal and Ocean Engineering $130,272$.

Gilleland, E., Brown, B. G., and C.M. Ammann, 2013. Spatial extreme value analysis to project extremes of large-scale indicators for severe weather. Submitted to Environmentrics.

Haver, S., and K. Nyhus, 1986. A wave climate description for long term response calculations. Proc. $5^{\text {th }}$ OMAE Symp. IV, 2734.

Heffernan, J. E., and J.A. Tawn, 2004. A conditional approach for multivariate extreme values. J. R. Statist. Soc. B 66, 497.

Jonathan, P., and K. C. Ewans, 2007. The effect of directionality on extreme wave design criteria. Ocean Engineering, 34, 1977-1994.

Jonathan, P., and K.C. Ewans, 2011. Modelling the seasonality of extreme waves in the Gulf of Mexico. ASME J. Offshore Mech. Arct. Eng. 133:021104.

Jonathan, P., and K. C. Ewans, 2013. Statistical modelling of extreme ocean environments with implications for marine design : a review. Ocean Engineering, 62, 91-109.

Jonathan, P., Ewans, K.C., and G. Z. Forristall, 2008: Statistical estimation of extreme ocean environments: The requirement for modelling directionality and other covariate effects, Ocean Engineering, 35, 1211-1225.

Jonathan, P., Ewans, K. C., Randell, D., 2013. Joint modelling of environmental parameters for extreme sea states incorporating covariate effects. Coastal Engineering 79, 22-31.

Jonathan, P., Randell, D., Wu, Y., and K. Ewans, 2014: Return level estimation from non-stationary spatial data exhibiting multidimensional covariate effects. Ocean Engineering (Submitted, preprint at www.lancs.ac.uk/ jonathan)

Jonathan, P., Ewans, K., and D. Randell, 2014: Non-stationary conditional extremes of northern North Sea storm characteristics. Environmetrics (In press, preprint at www.lancs.ac.uk/ jonathan).

Keef, C., Tawn, J., and C. Svensson, 2009. Spatial risk assessment for extreme river flows. J. Roy. Statist. Soc. C 58, 601-618.

Keef, C., Papastathopoulos, I., and J.A. Tawn, 2013a. Estimation of the conditional distribution of a vector variable given that one of its components is large: additional constraints for the Heffernan and Tawn model. J. Mult. Anal. 115, 396-404.

Keef, C., Tawn, J. A., R. Lamb, 2013b. Estimating the probability of widespread flood events. Environmetrics 24, $13-21$.

Kyselý, J., Picek, J., and R. Beranová, 2010. Estimating extremes in climate change simulations using the peaks-over- 
threshold method with a non-stationary threshold. Global and Planetary Change 72, 55-68.

Mendez, F. J., Menendez, M., Luceno, A., Medina, R., and N.E. Graham, 2008. Seasonality and duration in extreme value distributions of significant wave height. Ocean Eng. 35, 131-138.

Northrop, P., and P. Jonathan, 2011. Threshold modelling of spatially-dependent non-stationary extremes with application to hurricane-induced wave heights. Environmetrics 22, 799-809.

Ruggiero, P., Komar, P. D., and J.C. Allan, J. C., 2010. Increasing wave heights and extreme value projections: The wave climate of the U.S. pacific northwest. Coastal Eng. 57, 539-522.

Winterstein, S. R., Ude, T. C., Cornell, C. A., Bjerager, P., and S. Haver, 1993. Environmental parameters for extreme response: Inverse Form with omission factors. In: Proc. $6^{\text {th }}$ Int. Conf. on Structural Safety and Reliability, Innsbruck, Austria.

Winterstein, S. R., Jha, A. K., and S. Kumar, S., 1999. Reliability of floating structures: extreme response and load factor design. Journal of Waterway, Port, Coastal, and Ocean Engineering 125, 163-169. 